\title{
Corela
}

Cognition, représentation, langage

HS-1 | 2005

Colloque AFLS

\section{Codage phonologique et boucle articulatoire en mémoire de travail}

Un support pour la facilitation de l'accès à l'oral et à la lecture pour des publics débutants en français langue étrangère

\section{Michel Billières}

\section{(2) OpenEdition}

Journals

Édition électronique

URL : http://journals.openedition.org/corela/1110

DOI : 10.4000/corela. 1110

ISSN : 1638-573X

Éditeur

Cercle linguistique du Centre et de l'Ouest - CerLICO

Référence électronique

Michel Billières, «Codage phonologique et boucle articulatoire en mémoire de travail », Corela [En

ligne], HS-1 | 2005, mis en ligne le 16 février 2005, consulté le 30 avril 2019. URL : http://

journals.openedition.org/corela/1110; DOI : 10.4000/corela.1110

Ce document a été généré automatiquement le 30 avril 2019

\section{(c) (i) (3) (2)}

Corela - cognition, représentation, langage est mis à disposition selon les termes de la licence Creative Commons Attribution - Pas d'Utilisation Commerciale - Partage dans les Mêmes Conditions 4.0 International. 


\section{Codage phonologique et boucle articulatoire en mémoire de travail}

Un support pour la facilitation de l'accès à l'oral et à la lecture pour des publics débutants en français langue étrangère

\section{Michel Billières}

\section{Introduction}

1 La problématique du passage à l'écrit est complètement évacuée par la didactique du français langue étrangère (FLE) depuis l'avènement des approches communicatives au début des années 80. Dans la période antérieure, le courant structuro-global audio-visuel (SGAV) avait beaucoup réfléchi sur la question du passage à l'écrit dans les années 60-70. Il préconisait de travailler d'abord la compétence orale (écouter, puis parler) et de ne passer à l'écrit (lire, puis écrire) qu'au moment où l'élève avait déjà acquis une certaine aisance à l'oral, notamment grâce à un travail audio-phonatoire soutenu par l'utilisation de la méthode verbo-tonale d'intégration phonétique (Renard, 2002).

2 Actuellement, toutes les méthodes prévoient des activités de lecture dès les tous débuts de l'apprentissage linguistique. Les méthodes FLE pour débutants visent à installer très tôt une "rentabilité communicative » en répondant aux attentes communicationnelles des apprenants : se présenter, parler de soi, se situer dans l'espace, etc. Les documents variés -sonores, visuels, écrits- sont destinés à favoriser la prise de parole et l'interactivité. Il s'instaure un va-et-vient continuel entre pratiques orales et lecture de documents ou de petits textes. La lecture est censée prolonger certaines activités orales, préciser des points restants obscurs, permettre d'appréhender certains détails ou difficultés contenus dans les dialogues, offrir une plus grande autonomie à l'élève.

3 La didactique du FLE est essentiellement fondée sur une approche globale la lecture, apparue dans le courant des année 70, et qui privilégie la construction du sens. De fait, l'apprenant débutant en FLE est très tôt invité à mettre en œuvre ses compétences textuelles à la faveur d'activités favorisant des lectures interactives. Il semble aller de soi 
que ces compétences de haut niveau sont naturellement transférables telles quelles si l'élève est un bon lecteur dans sa langue maternelle. Ce dernier argument permet également de considérer qu'il s'accoutumera rapidement au système graphique du français. Ceci paraît évident pour les lecteurs dont les systèmes d'écriture obéissent aux mêmes principes de codage, comme les alphabets latin et cyrillique fondés sur la correspondance phonème-graphème. On soupçonne que les apprenants dont les alphabets sont fondés sur des représentations syllabiques ou idéographiques vont rencontrer de plus grandes difficultés mais, à notre connaissance, il n'y a guère de propositions didactiques afin de venir en aide à ces personnes. Tout au plus certaines méthodes de FLE pour débutants proposent-elles quelques activités de découverte des lettres de l'alphabet latin.

Cette contribution aborde le problème de la perception et du traitement des indices linguistiques sonores et scripturaux présentés par l'enseignant à des apprenants débutants complets en FLE. Elle ne traite pas de la question de la compréhension mais s'intéresse aux processus primaires de reconnaissance et d'identification des représentations linguistiques qui sont à l'origine du sens. La présentation des faits sous l'angle psycholinguistique nous permet de défendre la position suivante sur le plan didactique : l'introduction simultanée et sans la moindre précaution d'activités orales et de lecture en langue étrangère n'est pas une solution facilitante pour la plupart des élèves débutants ; elle ne permet pas de les aider à combler des déficits naturels dus au passage à un autre système linguistique.

5 A l'oral, l'élève est victime du phénomène de surdité phonologique. Il perçoit les sonorités $\mathrm{du}$ français -rythme, intonation, sons- sur la base du système phonique de sa langue maternelle. En outre, il segmente le flux verbal en se fondant sur ses habitudes linguistiques. A l'écrit, un processus de recodage phonologique semble automatiquement activé chez un lecteur non expert lors de la lecture silencieuse et à haute voix. Tout se passe comme si le lecteur devait passer par la prononciation des mots pour avoir accès à leurs représentations orthographiques. Ce fait fondamental est passé sous silence dans la didactique du FLE.

6 Notre démonstration se fera en trois moments. Tout d'abord, nous soulignerons qu'en langue maternelle un bon lecteur manifeste une conscience phonologique dont le fondement se trouve dans le codage correct de l'information phonologique. Ensuite, nous verrons qu'un codage phonologique adéquat en lecture garantit un meilleur accès aux unités lexicales. Notre troisième partie sera consacrée à la façon de contribuer à une meilleure perception de certaines unités prosodiques par les apprenants débutants en FLE. L'intégration progressive des marques prosodiques grâce à un entraînement audiophonatoire préalable contribue à l'émergence d'une conscience phonologique de nature à aider les débutants étrangers dans les activités de lecture en français.

\section{L'importance des représentations phonologiques pendant la lecture}

7 De très nombreuses recherches sur les étapes d'apprentissage de la lecture par les enfants, menées sur plusieurs langues, attestent de l'importance des concepts de conscience et de codage phonologiques, indispensables pour devenir un lecteur expert. 


\subsection{La conscience phonologique}

8 Le langage écrit constitue un moyen de transcrire le langage parlé. Toute écriture est plus ou moins phono-graphique. Les formes phonologiques permettant de transcrire les unités de sens sont présentes dans tous les systèmes d'écriture. C'est l'unité sonore représentée dans les symboles qui varie selon le type d'écriture. Une écriture logographique (chinois) privilégie le mot ou le morphème. Une écriture syllabique, celle du japonais, se fonde sur la syllabe ou la more.

9 Le principe d'un système d'écriture alphabétique est de représenter les unités phonémiques de l'oral par des graphèmes. Le lecteur doit relier ces unités orales à leur transcription graphique. Les conventions grapho-phonologiques des langues à système alphabétique diffèrent grandement en fonction des règles orthographiques propres à chacune d'elles. Le système orthographique de langues telles que l'allemand, l'italien ou l'espagnol est superficiel car il reflète assez fidèlement la phonologie de surface. La relation entre phonèmes et graphèmes est bi-univoque. Cette relation est au contraire particulièrement instable pour l'anglais et le français dont les systèmes orthographiques sont profonds ou opaques car ils représentent simultanément la langue aux niveaux phonologique, morphologique et syntaxique.

10 Le développement d'une conscience phonologique est indispensable pour devenir un lecteur expert. Les recherches menées sur les langues à système d'écriture alphabétique sont unanimes sur ce point. La conscience phonologique est la capacité d'identifier les composantes phonologiques des unités linguistiques et de les manipuler délibérément. Elle est à l'origine de la capacité de segmenter l'oral en unité non significatives. Une personne éprouvant des difficultés à analyser la structure phonologique de la langue orale ou ne parvenant pas à établir des liens entre les systèmes écrit et oral se trouve dans l'incapacité d'accéder au principe même du système alphabétique et rencontre des difficultés majeures dans la reconnaissance des mots écrits.

On considère quatre niveaux de conscience phonologique apparaissant à différentes strates de l'acquisition linguistique chez le jeune enfant :

- la conscience des chaînes phonologiques. Elle est liée à la sensibilisation aux facteurs prosodiques et se manifeste très tôt ;

- la capacité à repérer les rimes des mots parlés sans prêter attention à la signification ;

- la conscience syllabique qui apparaît précocement à la faveur du développement de la langue orale. La structure syllabique et la longueur des mots jouent un grand rôle dans l'action de découpage en syllabes ;

- la conscience phomémique est le niveau le plus haut. Elle est caractérisée par le fait que l'enfant est capable, dans un premier temps, de discriminer deux sons en disant s'ils sont semblables ou différents, puis est à même dans un deuxième temps, de nommer explicitement les sons entendus. La conscience phonémique est une habileté métaphonologique essentielle. Elle se développe pendant l'apprentissage du système alphabétique dont le principe est de pouvoir décomposer les mots en phonèmes.

11 La conscience phonologique et l'apprentissage de la lecture se développent en interaction et se renforcent mutuellement. 


\subsection{Le codage phonologique}

12 En situation de lecture silencieuse ou à haute voix d'une écriture alphabétique, le codage phonologique est un processus qui intervient automatiquement et de manière irrépressible. L'information phonologique des mots écrits est susceptible d'être récupérée de deux façons :

- par adressage. Le mot est reconnu comme une configuration orthographique particulière, sa prononciation est ensuite récupérée à une adresse en mémoire -phonologie lexicale- ;

- par assemblage. Au fur et à mesure de la lecture, la représentation orthographique est segmentée en segments auxquels sont associés les segments phonologiques correspondants -phonologie pré lexicale-.

Un codage opéré à partir d'une information phonologique adéquate concourt à favoriser certains traitements visant à la compréhension :

- il favorise le maintien des représentations en mémoire de travail ;

- il assure un meilleur repérage de la configuration prosodique ;

- il garantit une meilleure résistances aux interférances entre les représentations activées en mémoire de travail et celles en mémoire à long terme ;

- il constitue une aide supplémentaire à la vitesse et à la précision de l'activation orthographique dans l'identification des mots connus.

\section{La reconnaissance et l'identification des représentations linguistiques}

13 La majorité des modèles psycholinguistiques de perception, compréhension et production du langage est de type traitement de l'information; celle-ci est organisée en une suite d'étapes distinctes -variables selon les modèles- dans un ordre chronologique.

\subsection{La notion de lexique mental}

14 L'apprenant veut comprendre ce qu'il entend ou lit. Pour ce faire, il s'efforce de repérer les mots qui constituent une unité de prédilection de part leur poids psychologique. L'accès aux mots est une opération cognitive éminemment complexe. Dans cette partie, nous nous intéresserons au faits cognitifs impliqués en langue maternelle. Ceci nous permettra de mieux cerner les déficits de débutants en langue étrangère dans la partie suivante consacrée au renforcement de l'information phonologique.

La recherche des mots s'effectue en accédant au lexique mental. Ce lexique interne, postulé par de nombreux modèles sériels, est une structure mentale hypothétique de la mémoire à long terme. Il permet de stocker et de récupérer toutes les informations phonologiques, orthographiques, morphologiques, syntaxiques, sémantiques- des mots qu'ils soient présentés auditivement, visuellement, ou prononcés. Le lexique mental regroupe un certain nombre de paramètres formels dépendant de la modalité sensorielle sollicitée -auditive ou visuelle-: longueur, fréquence, régularité orthographique, homophonie, similarité phonétique, visuelle ou orthographique.

16 Le concept de lexique mental représente le trait d'union entre la perception de la parole (niveau acoustico-phonétique) et les niveaux supérieurs du traitement cognitif (niveau linguistique). "Le lexique mental permet la mise en relation de l'information sensorielle (le 
stimulus mot) et de la représentation lexicale. Il y a d'un côté les représentations du lexique mental, de l'autre le stimulus mot, et entre les deux les procédures permettant d'atteindre ou d'activer les représentations à partir du stimulus » (Babin, 1998, 5).

\subsection{Les opérations en amont de l'accès au lexique}

\subsection{1. la mémoire de travail comme centre de gestion des représentations}

Les informations linguistiques auditives et visuelles perçues, directement issues de la stimulation, doivent être d'abord reconnues avant d'être identifiées. Les processus de traitement ascendant assurant leur traitement dynamique sont déterminés étroitement par les caractéristiques sensorielles et leur organisation. Ces processus de bas niveau sont automatiques, extrêmement rapides, irrépressibles, et inconscients. Une forme auditive ou scripturale n'a pas de signification à l'issue des étapes de reconnaissance. Elle ne peut être identifiée que quand il y a appariement avec une représentation lexicale lui correspondant.

Cette identification s'opère pendant que l'information sensorielle est temporairement hébergée en mémoire de travail (MDT). Dans le modèle de Baddeley, la MDT est constituée d'un mécanisme passif de stockage des informations et d'un mécanisme actif destiné à les traiter. Des traitements de haut niveau interviennent lors des phases d'identification. Ils sont également très rapides, automatiques et inconscients, mais peuvent parfois faire l'objet d'un contrôle. Ces traitements descendants prennent en compte les connaissances et les intentions du sujet.

En modalité auditive, les sonorités sont prise en charge par un système esclave appelé boucle phonologique. Elle fonctionne à la manière d'un magnétophone très sensible aux caractéristiques physiques des sonorités. Une composante de stockage passif (enregistrement) maintient les informations verbales ou prononçables en retardant l'oubli. Une composante active, la boucle articulatoire (lecture), convoque la trace phonologique encore disponible par un mécanisme de répétition mentale, le plus souvent implicite.

20 En modalité visuelle, les modèles actuels d'acquisition de la lecture soulignent le rôle central de l'information phonologique dans l'identification visuelle des mots écrits. Le lecteur voyant un mot doit le traduire en sons en effectuant une opération de recodage phonologique. Cette répétition mentale de la prononciation du mot lui permet d'accéder à sa signification. "Le recours au code phonologique est l'un des fondements de la lecture efficace " (Golder et Gaonac'h, 1998, 38).

Il existe 3 positions dominantes concernant le codage phonologique :

- la théorie d'accès direct, selon laquelle sur le codage orthographique est important, la phonologie n'étant pas convoquée. Ce point du vue est maintenant quasiment abandonné ;

- la théorie de la médiation phonologique, pour laquelle seul le codage phonologique est important, l'orthographe ne jouant aucun rôle. Ce point de vue est maintenant considéré comme extrêmiste ;

- la théorie des deux routes, pour qui le code orthographique est dominant, le code phonologique intervenant de façon optionnelle dans le cas de mots difficiles, rares, ou inconnus; Grainger qui suppose une action conjointe des deux codes, phonologique et 
orthographique, sans domination de l'un ou de l'autre. Ces deux dernières approches paraissent les plus réalistes.

\subsubsection{La syllabe, comme unité infra-lexicale capitale}

considérer "... que l'analyse segmentale de la parole repose sur l'établissement d'une unité de niveau suprasegmental: la syllabe» (Segui et Ferrand, 2000, 173). Des spécialistes de la prosodie du français abondent dans le même sens : «la syllabe, potentiellement porteuse de l'accent, apparaît comme l'unité minimale de perception rythmique. Elle constitue en effet l'unité de base dans la segmentation de la parole et l'unité clé d'accès au lexique " (Lacheret-Dujour et Beaugendre, 1999, 45). développement comportemental des enfants apprentis lecteurs d'un système d'écriture alphabétique ${ }^{1}$. Les enfants débutant l'apprentissage de la lecture recourent systématiquement à un traitement phonologique qui s'appuie d'abord sur des unités grapho-phonémiques puis sur des unités grapho-syllabiques. Ces dernières représentent un moindre coût cognitif. Ces deux procédures de conversion sont systématiquement utilisées pendant un temps. L'enfant acquérant un meilleur degré d'expertise a recours à la procédure phonologique syllabique pour les mots familiers ou courts et utilise la procédure grapho-phonémique quand il est en présence de mots inconnus, rares ou longs. Devenu bon lecteur, l'enfant recourt à une procédure orthographique et non plus phonologique pour traiter les mots familiers et réserve la procédure phonologique de type syllabique pour traiter les mots rares. "Ainsi, le traitement syllabique résulterait de la capacité à établir des liens entre des séquences de lettres et la prononciation de parties de mots, c'est-à-dire à élaborer des représentations ortho-phonologiques » (Ecalle et Magnan, ibid., 155).

De très nombreuses expériences de chronométrie mentale attestent du rôle essentiel de la syllabe dans l'identification visuelle des mots écrits en lecture silencieuse et à haute voix. Les lecteurs groupent les lettres en syllabes qui constituent des unités fondamentales de lecture. Dans les systèmes de lecture alphabétique, les mots ne constituent pas qu'une 
simple suite de phonèmes ou de lettres. Ils s'organisent autour d'un groupe plus grand que le phonème mais moins grand que la syllabe. Les deux unités primaires de la syllabe sont l'attaque et la rime qui semblent jouer un rôle important dans la lecture (Ferrand, 2002, 69-70). Avec une restriction importante : les habitudes de segmentation en lecture diffèrent selon les langues, notamment au niveau des unités infra-lexicales (graphèmes, syllabes, morphèmes).

\section{Le renforcement de l'information phonologique en langue étrangère}

\subsection{L'action sur le processus de mémorisation}

L'apprenant débutant l'étude du français s'attache souvent aux aspects les plus formels d'un code linguistique dont les représentations ne sont pas installées en mémoire et courent le risque d'être fréquemment erronées ou faussées. La manipulation des symboles du langage de la langue étrangère engendre des déficits dans la mise en œuvre d'un certain nombre de processus pour le codage verbal ainsi que pour l'accès et le maintien en mémoire. Certains automatismes font défaut, d'autres durent trop longtemps. L'élève perd du temps et ne parvient pas à gérer les diverses informations à différents niveaux linguistiques.

Parmi toutes ces informations, celles relatives aux aspects phonologiques jouent un rôle capital. Elles assurent un meilleur accès à la reconnaissance et à l'identification des mots entendus ou lus. Elles doivent prioritairement être travaillées à l'oral qui constitue leur modalité naturelle.

Est-il possible pour l'enseignant d'agir sur les sonorités parolières et d'en modifier les représentations dans le chef de l'apprenant? On répondra positivement en faisant remarquer que

- sur le plan didactique, la méthode verbo-tonale, quand elle est utilisée par un professeur expert, améliore sensiblement la performance phonétique de nombreux élèves en FLE (Intravaia, 2000) ;

- sur le plan psycholinguistique, l'utilisation en temps réel des diverses procédures de correction proposées en verbo-tonale ${ }^{2}$ trouve une légitimité supplémentaire en s'appuyant sur les résultats d'expérimentations portant sur le fonctionnement de la mémoire de travail (modèle de Baddeley) et la nature des unités que celle-ci doit traiter.

31 La MDT constitue un lieu temporaire de rétention et de traitement actif des données. L'information verbale codée phonologiquement est stockée au niveau de la boucle phonologique. Elle est maintenue active grâce au processus d'autorépétition mentale. L'apprenant peut alors être confronté à une série de déficits :

- Le maintien du matériel verbal peut être affecté par une carence de la boucle phonologique ou du processus d'autorépétition en raison du confit entre ces représentations activées comparées avec celles présentes en mémoire à long terme (MLT) ; ces dernières sont en effet conformes aux patrons phonologiques de la langue maternelle.

- L'élève doit traiter simultanément l'information qu'il vient de stocker et celle qu'il est en train d'entendre. Il lui faut relier l'ensemble de ces données avec celles qu'il possède (éventuellement) en MLT afin de saisir le sens de l'énoncé. Ce désir de comprendre la parole étrangère est irrépressible et peut accroître la surdité phonologique. L’apprenant mobilise 
toutes ses connaissances et néglige de prêter attention aux sonorités qui sont le simple vecteur de la parole.

- L'administrateur central, composante attentionnelle de la MDT établissant notamment les liaisons avec la MLT, peut être perturbé par l'intrusion de paramètres verbaux inhabituels qu'il ne parvient pas à gérer dans les délais impartis par le système de traitement. De nombreuses expériences attestent que la boucle phonologique maintient l'information sonore active pendant 1, 5 seconde. Cette information est ensuite " effacée »; il faut laisser la place à la suite de l'information entrante en MDT.

Ce délai de 1, 5 seconde est suffisant pour permettre à l'enseignant d'agir sur les qualités physiques des stimuli phonologiques. En utilisant tel ou tel procédé en fonction de l'erreur de production commise par l'apprenant (qui prononce $[\mathrm{u}]$ au lieu de $[\mathrm{y}]$ par exemple), le professeur intervient sur la perception sensorielle du stimulus dont il modifie certaines propriétés. Cette représentation présente en MDT est "comparée » avec les représentations antérieures disponibles dans la mémoire permanente du sujet. Cette comparaison engendre un processus de transformation. En effet, une représentation est par nature

- précaire, car tout changement dans la situation peut provoquer sa modification;

- transitoire, car elle est remplacée par une autre représentation une fois la tâche achevée

C'est toujours une nouvelle représentation qui va en MLT. La progression est liée à cette faculté de l'esprit humain. L'apprenant répète des stimuli sonores présentés par l'enseignant de façon optimale en fonction de l'erreur commise. Une action est directement exercée à la source de l'information sensorielle, antérieurement à l'étape cruciale de son traitement en MDT La perception auditive de l'apprenant s'affine au fur et à mesure de l'entraînement phonétique. Le codage phonologique s'effectue en intégrant graduellement les spécificités physiques des sonorités parolières de la langue étrangère. De nouvelles représentations sonores intègrent la MLT. Elles influencent la perception en installant progressivement un nouvel espace sonore familier, donc de plus en plus prévisible et prédictible pour l'apprenant.

\subsection{Les unités sonores sur lesquelles porte l'action remédiatrice}

La correction phonétique doit permettre d'améliorer la production incorrecte des voyelles et des consonnes. Cette conception traditionnelle de la phonétique corrective est héritée du structuralisme. Le phonème est l'unité pivot de l'acte de remédiation. Une bonne prononciation permet de lever des ambiguïtés au niveau du code linguistique. De fait, tous les manuels de phonétique corrective insistent sur la façon d'éradiquer les erreurs produites sur les segments.

Les segments vocaliques et consonantiques ne sont pas produits isolément dans l'activité parolière. Ils s'insèrent naturellement dans un cadre rythmico-intonatif. Les marques prosodiques exercent naturellement une certaine influence sur la qualité des unités segmentales ${ }^{3}$. Partant, la priorité est toujours accordée à la prosodie, que ce soit pour intervenir sur un rythme ou une intonation défectueux ou pour corriger une voyelle ou une consonne mal réalisées (Billières, $1993 ; 2002 ; 2003$ ).

Dans cette perspective, la syllabe, dont nous avons vu l'importance dans les processus de segmentation à l'oral et à l'écrit (cf. 3.2.2.), constitue une unité particulièrement opératoire de par ses caractéristiques. Unité fondamentale en perception, elle est le lieu 
de la variation segmentale, de la coarticulation et des assimilations; elle reflète également les divers degrés d'accentuation. Sa durée moyenne se situe dans les limites des capacités de stockage du système sensoriel. La boucle phonologique en MDT peut traiter des blocs de 3 à 4 syllabes. Ceci correspond au nombre limité d'éléments dont elle peut assurer la rétention et l'activation pendant 1,5 seconde. Et ceci est conforme aux productions de l'oral spontané du français où dominent des unités rythmiques très courtes dont la grande majorité est formée autour de 3 syllabes ${ }^{4}$.

La concordance des chiffres s'explique par le fait que les capacités cognitives du système de traitement langagier de l'humain présentent des limites ; l'individu ne peut traiter à la fois que de petits groupes d'éléments. Il doit d'abord les structurer; ce n'est qu'ensuite qu'il peut construire une représentation sémantique unique intégrée prenant en compte la totalité des informations. Il est plus facile de traiter des structures courtes les unes après les autres. Leur synthèse aboutit à une interprétation du sens global du message à partir des données déjà traitées et en fonction des connaissances de l'individu (Gineste et Le $\mathrm{Ny}, 2002,101$ suiv.).

Ceci précisé, quels sont les points à retenir pour exercer une action efficace au niveau de la boucle phonologique et, partant, du mécanisme de récapitulation articulatoire?

Le français offre un rythme syllabique très homogène. Les structures syllabiques sont régulières et les frontières entre syllabes nettement marquées. Du fait de la syllabation ouverte dominante, la voyelle a un poids psychologique important. Elle est vraiment centre de syllabe; son articulation est nette et tenue pendant toute la durée de son émission. Il semble que la consonne ait besoin d'un appui vocalique pour exister; dès sa naissance, elle vient s'agglutiner étroitement à la voyelle suivante. Ceci peut être illustré de la manière suivante :

Syllabation ouverte > anticipation vocalique > enchaînement consonantique.

41 L'enseignant a tout intérêt à proposer systématiquement des unités rythmiques très brèves en début d'apprentissage phonétique. Et ce pour les 2 raisons mentionnées en supra et que nous rappelons: l'oral spontané est ce qui est théoriquement visé par les méthodes de FLE (cf. les enregistrements dits "authentiques»); des blocs phoniques courts sont plus faciles à gérer par l'esprit humain.

42 A titre d'exemple, l'énoncé Bonjour monsieur. Vous allez bien? peut être segmenté en 2 groupes de 4 syllabes. Ceci correspond aux limites du système de traitement en MDT. Avec un débutant non entraîné à percevoir les sonorités du français, il sera plus rentable de segmenter en Bonjour / monsieur / vous allez / bien, soit 2 / 2 / 3 / 1 syllabes. La boucle phonologique peut prendre ces blocs successifs en charge avec un plus grand confort. Il y a moins d'éléments à traiter par bloc; le temps imparti à chacun d'eux est plus important 5 .

Ce principe de découpage en brèves unités successives vaut aussi quand 2 groupes sont quantitativement déséquilibrés comme dans l'exemple Au fait $\neq t u$ n'aurais pas $v u$ mon livre? ( $\neq$ indique une pause) où nous avons 2 unités consécutives de 2 et 7 syllabes. Il faudra découper en 4 blocs de 2 / 3 / 2 / 2 syllabes au fait / tu n'aurais / pas vu / mon livre, ou en 3 blocs de 2 / 3 / 3 syllabes au fait / tu n'aurais pas / vu mon livre, qui ne poseront pas de problème de restitution à un débutant victime de surdité phonologique. La sensibilisation à la syllabation ouverte est beaucoup plus facile à travailler avec de petits groupes rythmiques. L'enseignant doit aussi savoir tirer profit de la présence de tout accent, primaire ou secondaire. Les syllabes concernées par ces divers degrés 
d'accentuation sont autant de proéminences rythmiques. Elles constituent d'utiles points de repère et aident à dynamiser la propagation de bloc phonique en bloc phonique.

Faire lire les élèves débutants en français dès le premier cours peut se concevoir dans une optique pédagogique. Il s'agit de leur donner tous les moyens d'accès à la langue. Sur le plan psycholinguistique, cette approche est très coûteuse car les processus mobilisés sont loin d'être automatisés comme c'est le cas pour la langue maternelle.

A première vue, certaines commodités s'offrent à l'apprenant. à l'écrit. La partition en mots est fournie directement et instantanément. Il peut lire à la vitesse qu'il désire ; il a également le loisir de revenir en arrière pour procéder à des vérifications. Mais la lecture est aussi source de pièges insidieux. Rappelons qu'un lecteur non expert active un processus de recodage phonologique agissant sur la boucle de récapitulation articulatoire de la MDT en langue maternelle. Le même phénomène intervient en langue étrangère, mais aggravé par toutes les lacunes langagières et les déficits cognitifs dus à l'utilisation d'un autre code linguistique. Les mécanismes de correspondance grapho-phonologique ainsi que ceux de la segmentation des unités infra lexicales sont toujours remis en cause lors du passage à une langue autre que maternelle. L'élève qui entreprend une activité de lecture déchiffre les lettres et identifie les mots avec plus ou moins de difficulté. Il est en proie au phénomène de surdité phonologique. Il passe nécessairement par les sonorités de sa langue maternelle, ou d'autres langues déjà connues, dans l'activité de récapitulation articulatoire accompagnant sa lecture, Cette stratégie compensatrice lui permet de gagner du temps et de ne pas être complètement submergé par l'abondance d'informations inhabituelles. D'autant plus que ces informations sonores déformées ne remettent pas fondamentalement en cause la compréhension globale. Elles constituent des points de détail pouvant constituer une gêne plus ou moins ressentie. Toutes les ressources conceptuelles de l'apprenant sont mobilisées pour accéder à une compréhension aussi correcte que possible du texte lu sans perdre de temps dans l'identification et la planification articulatoire de sonorités. Celles-ci ne sont jamais que le simple vecteur physique conduisant à la signification. Dans tous les cas, c'est la nouvelle langue étudiée qui fait les frais de ces carences audio-perceptives qui sont négligées en début d'apprentissage.

Le monde sonore paraît truffé d'embûches autrement plus pernicieuses. A l'audition, les frontières physiques des mots ne sont pas marquées dans le signal de parole. L'élève doit lui-même procéder à leur repérage. L'information étant présentée linéairement, l'apprenant n'a aucune possibilité de retour en arrière. Il est en outre tributaire de la vitesse de parole du locuteur.

Une pratique audio-phonatoire bien menée doit permettre à beaucoup d'apprenants étrangers d'améliorer leur compétence phonétique. En d'autres termes, leur surdité phonologique est entamée et ils sont plus sensibles aux spécificités prosodiques et segmentales du français. Ils sont donc davantage à même de pouvoir accéder à une conscience phonologique dont la maîtrise est indispensable pour devenir un bon lecteur en français. 


\section{BIBLIOGRAPHIE}

Babin, J.-P. 1998. Lexique mental et morphologie lexicale Bern, Peter Lang, 257 p.

Baddeley, A. 1993. La Mémoire humaine. Théorie et pratique. Grenoble, Presses Universitaires de Grenoble, $547 \mathrm{p}$

Billieres, M. 2002. " Des activités de classe aux activités cognitives en phonétique corrective » Actes du XIIIème colloque international SGAV, Cahiers du CISL, 2003, Université de Toulouse-Le Mirail, 12-14 septembre 2002, p. 71-87

Billieres, M. 2002 « Le corps en phonétique corrective ", dans : Apprentissage d'une langue étrangère/seconde 2. La Phonétique verbo-tonale. (R. Renard, coord.) Bruxelles, De Boeck Université, 333 p. (coll. Pédagogies en Développement) 37-70

Billieres, M. 1999 « Psycholinguistique et méthodologie verbo-tonale » Revue Parole, $\mathrm{n}^{\circ}$ 11-12, p. $173-198$

Billieres, M.1993 « Théorie et pratique du rythme parolier en phonétique corrective », Cahiers du Centre Interdisciplinaire des Sciences du Langage, $\mathrm{n}^{\circ}$ 9, Université de Toulouse-le Mirail, p. 3-32

Ecalle, J. ; Magnan, A. 2002. L'Apprentissage de la lecture. Fonctionnement et développement cognitifs, Armand Colin, $320 \mathrm{p}$

Fayol, M. ; Gombert, J.-E. ; Lecocq, P. et al. 1992. Psychologie cognitive de la lecture, PUF, 288 p

Ferrand, L. 2001. Cognition et lecture. Processus de base de la reconnaissance des mots écrits chez l'adulte Bruxelles, De Boeck Université, 381 p

Gaonac'h, D. (coord.), 1990. Acquisition et utilisation d'une langue étrangère Le Français dans le Monde, série « recherches et applications », février-mars 1990

Gaonac'h, D. 1987. Théories d'apprentissage et acquisition d'une langue étrangère, Paris, Crédif-Hatier, $239 \mathrm{p}$

Gaonac'h, D. ; Fayol, M. 2003. Aider les élèves à comprendre du texte au multimédia, Hachette éducation, $255 \mathrm{p}$

Gaonac'h, D. ; Larigauderie, P. 2000. Mémoire et fonctionnement cognitif, Armand Colin, 284 p.

Gineste, M.-D. ; Le Ny, J.-F. 2002. Psychologie cognitive du langage, Dunod, 170 p.

Golder, C. ; Gaonac'h, D. 1998. Lire et comprendre. Psychologie de la lecture, Hachette, 143 p

Intravaia, P. 2000. Formation des professeurs de langue en phonétique corrective. Le système verbo-tonal Didier Érudition, Paris et CIPA, Mons, 283 p (avec 4 CD)

Kolinsky, R. ; Morais, J. ; Segui, J. 1991. La Reconnaissance des mots dans les différentes modalités sensorielles : études de psycholinguistique cognitive, PUF, 1991, $320 \mathrm{p}$

Lacheret-Dujour, A. ; Beaugendre, F. 1999. La Prosodie du français, CNRS Éditions, $354 \mathrm{p}$

Llorca, R. 1987. Éléments d'analyse du rythme de la parole en français Thèse NR, Université de Franche-Comté, Besançon, $378 \mathrm{p}$

Morais, J. 1999. L'Art de lire, Odile Jacob, 362 p (coll. Opus) 
Renard, R. (coord.), 2002. Apprentissage d'une langue étrangère/seconde 2. La phonétique verbo-tonale Bruxelles, De Boeck Université, 333 p (coll. Pédagogies en développement)

Romdhane, M. N. ; Gombert, J.-E. ; Belajouza, M. (dir.), 2003. L'Apprentissage de la lecture. Perspectives comparatives. Presses Universitaires de Rennes, $386 \mathrm{p}$

Segui, J. ; Ferrand, L. 2000. Leçons de parole, Odile-Jacob, 248 p

Wioland, F. 1984. « Organisation temporelle des structures rythmiques du français parlé » Bulletin des rencontres régionales de linguistique, Lausanne, pp. 293-322

\section{NOTES}

1. Cf. la recension de la littérature sur ce thème dans Ecalle et Magnan (2002, p. 117-155). Dans ce paragraphe, nous suivons l'argumentation de ces auteurs p. 154-155.

2. La MVT propose plusieurs procédés de correction, qui sont détaillés dans les ouvrages et articles de verbo-tonale indiqués en bibliographie, pour corriger une production sonore défaillante. Ces procédés de remédiation ont été mis au point, empiriquement et expérimentalement, dans les années 60-70. Les acquis de la psycholinguistique permettent au phonéticien didacticien d'améliorer sa pratique en intégrant des données sur le fonctionnement « interne » de l'apprenant en situation d'écoute en langue étrangère.

3. Par exemple, une intonation montante éclaircit naturellement le timbre des voyelles qui est assombri au contraire en creux intonatif. De même, l'intonation montante augmente la tension des consonnes, le phénomène inverse survenant en intonation descendante.

4. Pour la production, cf. le travail de Llorca (1987); pour la perception, cf. celui de Wioland 1984).

5. Pour un examen détaillé des procédures de correction à appliquer au rythme et à l'intonation, cf. Billières 1993 et 2002.

\section{RÉSUMÉS}

Les méthodes de français langue étrangère pour débutants proposent des activités de lecture dès les tous débuts de l'apprentissage. De nombreuses recherches en psycholinguistique établissent qu'un processus de récapitulation articulatoire est activé en mémoire de travail pendant la tâche de lecture. Le lecteur étranger fait appel aux sonorités de sa langue maternelle car il est victime du phénomène de surdité phonologique. La question de l'importance de la correction phonétique en tout début d'apprentissage se trouve posée une fois de plus. Cet article traite des unités sonores que l'élève convoque quand il lit. Le travail de correction phonétique proposé en situation orale est destiné à permettre leur meilleure reconnaissance, ce qui garantit un codage phonologique amélioré qui aura des conséquences positives sur l'activité de lecture.

From the outset Franch as a Foreign Language textbooks for beginners offer reeading activities. A lot of the research in psycholinguistic shows that the process articulatory loop is activated within the working memory while reading. The Foreign reader uses their mother tongue sounds because is a victim of the phonological deafness phenomenum. Once again, the importance of the 
phonetic correction during the early stages of language learning is questioned. The article presents the sounds that the student uses when he reads. Working on the phonetic correction orally will improve sound recognition garanteeing an improved phonetic code that in turn will have positiveconsequences on the reading activity.

INDEX

Mots-clés : apprentissage, français langue étrangère, lecture, mémoire, perception, prosodie, syllable, système verbotonal

\section{AUTEUR}

\section{MICHEL BILLIÈRES}

Université de Toulouse-Le Mirail, laboratoire Jacques-Lordat, EA 1941 